\title{
Migration, Legalität und Kriminalität: Rechtssoziologische Wissensdefizite im Schatten administrativen Wissens
}

\author{
Walter Fuchs • Katrin Kremmel • Andrea Kretschmann • Arno Pilgram
}

(C) Der/die Autor(en) 2016. Dieser Artikel ist eine Open-Access-Publikation.

Zusammenfassung Dieser Artikel zeigt Bereiche rechtssoziologischen Nichtwissens innerhalb der Migrationsforschung auf. Die Frage, was wir nicht wissen und weshalb diese Wissenslücken zustande kommen, wird mit gesellschaftlichen (Herrschafts- und Kontroll-)Praktiken in Zusammenhang gebracht. Hieraus leitet sich zum einen die Notwendigkeit einer kritischen Reflexion (impliziter) theoretischer Positionen in der Migrationsverwaltung ab, um davon ausgehend aktuelle Entwicklungen von Migrationskontrolle in den Blick zu nehmen. Zum anderen folgt daraus, die Spur jenes Wissens aufzunehmen, welches neben der enormen Fülle an administrativem Wissen ein Schattendasein führt. Es wird argumentiert, Defizitperspektiven, die MigrantInnen vorrangig als Objekte der Sicherheitsverwaltung begreifen, den Rücken zu kehren und stattdessen von MigrantInnen auch als ProduzentInnen von innerer Sicherheit und AkteurInnen in Konfliktlösungsprozessen auszugehen.

Schlüsselwörter Migration · Migrationskontrolle $\cdot$ Recht $\cdot$ Rechtsgebrauch · Kriminalisierung $\cdot$ Konfliktregelung

W. Fuchs $(\bowtie) \cdot$ A. Pilgram Institut für Rechts- und Kriminalsoziologie, Museumstrasse 5/12, 1070 Wien, Österreich E-Mail: walter.fuchs@irks.at
A. Pilgram
E-Mail: arno.pilgram@irks.at
K. Kremmel
E-Mail: katrin.kremmel@univie.ac.at
A. Kretschmann
Centre Marc Bloch, Humboldt-Universität, Berlin, Deutschland
E-Mail: kretschmann@cmb.hu-berlin.de

Institut für Kultur- und Sozialanthropologie, Universität Wien, Wien, Österreich 


\title{
Migration, Legality, and Crime: socio-legal knowledge gaps in the shadow of administrative knowledge
}

\begin{abstract}
This article aims to detect socio-legal knowledge gaps within migration studies. The questions of 'what we do not know', and how these knowledge gaps come into being, allow us to reflect on the relationship between knowledge on the one side and societal practices (of domination and control) on the other. We hence proceed to claim the necessity of ongoing inquiry into the (implicit) theoretical positions of the administration and control of migration processes and migrants. As a second endeavour we begin to search for 'the nescience that we don't know of'. Such an undertaking turns its back on theories promoting a conception of migrants as objects of control, rather to perceive migrants as producers of internal security and actors in processes of conflict resolution.
\end{abstract}

Keywords Migration · Migration Control · Law · Legal Practice · Criminalization · Conflict Resolution

\section{Einleitung: Zur Aufgabe, administratives Wissen und Polizeiwissen zu transzendieren}

Kaum ein soziales Phänomen ist stärker an rechtliche Vorgaben und administrative Praktiken gekoppelt als die internationale Migration. Ob bei der Frage nach Kontinuität und Wandel von Migrationsbewegungen und ihrer Steuerung oder der sozialen Position von MigrantInnen - immer ist das Soziale wesentlich durch Recht und seine Adaptionen durch Polizei und Verwaltung bestimmt. Gesetzgebung und Gesetzesvollzug durch die Exekutive und Judikative beeinflussen die Bedingungen, unter denen etwa Grenzen passiert werden, Arbeit aufgenommen wird oder politische Partizipation stattfindet. Ebenso bestimmen sie das Risiko von MigrantInnen, exkludiert, kontrolliert und sanktioniert zu werden, sowie deren Möglichkeit, Polizei, andere Behörden, Justiz und Recht auch für sich in Anspruch zu nehmen. Grundsätzlich ist Migrationsforschung dabei mit einem Gegenstand konfrontiert, der nicht nach disziplineigenen Kriterien ,,vorsortiert“ ist. Schließlich ist es der Staat, der Migration und MigrantInnen durch migrationspolitisches Zutun erst hervorbringt. Ohne staatliche Verwaltungseinheiten, die Menschen einem umgrenzten Territorium zuordnen, gibt es keine Verteidigung territorialer Grenzen staatlicher Souveränität oder auch eines ,Volkskörpers“, ohne von der Legislative für „Fremde“ geschaffene (Straf-)Tatbestände und deren Ahndung durch Exekutive und Justiz keine ,illegale“ Migration. Verbunden damit, liegt über der Existenz von Migration a priori der Vorbehalt eines besonderen Verwaltungs- und Regulierungsbedarfs, insofern auch eine Problemvermutung, die durch den beträchtlichen (Migrations-)Rechts- und Kontrollapparat praktisch und symbolisch unterstrichen wird und bestätigt scheint.

Das macht Migration zu einem Gegenstand der Rechts- und Kriminalsoziologie. Wissenslücken sehen wir jedoch weniger darin, akkumuliertes administratives und polizeiliches Praxiswissen aufzugreifen, zu systematisieren und die polizeiliche Migrationskontrolle zum „Migrationsmanagement“ (Cuttitta 2010) weiter zu 
entwickeln, wie dies, gerade in den traditionell staatsnahen, mit Recht und Politik befassten Wissenschaften, oft geschieht (vgl. Young 1986, S. 4). So hat das verstärkte Interesse migrationsregulatorischer Regime an Ergebnissen aus der Migrationswissenschaft gerade in den letzten Dekaden zu einer verstärkten Durchdringung von Migrations-(Grundlagen-)Forschung und „operativer Wissensproduktion“ geführt (Hess und Kasparek 2010, S. 14 f.) ${ }^{1}$. Eine ähnlich intensive, aber ungleich ältere Nähe zur Migrationsverwaltung kann auch die Kriminologie für sich beanspruchen. Sie versteht sich - historisch wie aktuell - überwiegend als Hilfswissenschaft von Polizei und Justiz. Es geht ihr im Wesentlichen um die Analyse alltagskrimineller wie auch besonderer, etwa organisiert krimineller oder terroristischer Risikopotenziale und um die Optimierung bestehender (staatlicher) Steuerungsinstrumente im Bereich des Kriminalrechts und der Polizei. Heinz Steinert beschrieb die „Reflexion des Selbstverständlichen“ (2008, S. 162) als zentrale Aufgabe sozialwissenschaftlicher Theorie. Viele jener staatsnahen Analysen jedoch, insofern sie von vornherein Problemstellungen und Zieldimensionen staatlicher Rationalitäten übernehmen, (re-) produzieren letztlich politische Kategorien wie „Fremder“, „Illegaler“, Grenze usw. (Georgi und Wagner 2009, S. 307), anstatt ihre Genese, Beschaffenheit und den gesellschaftlichen Umgang mit ihnen in den Blick zu nehmen. Ganz im Sinne Steinerts wie auch in der Tradition einer sich kritisch verstehenden Kriminologie halten wir es insofern für notwendig, die zentralen administrativen Kategorisierungen von MigrantInnen nach ihrer rechtlichen (Sonder-)Stellung bzw. nach der Illegalität ihres Handelns zu hinterfragen und mit diesen Kategorisierungen nicht auch die impliziten Defizit- und Problemperspektiven der Administration zu übernehmen. Eine kritische rechtssoziologische Forschung im Feld der Migration muss sich der Aufgabe stellen, derartige Zuschreibungen und ihre rechtlich-institutionellen Lösungsansätze als solche zu reflektieren, d. h. ihrerseits zu problematisieren.

Zwei Forschungsdesiderate leiten sich hieraus ab:

1. Bedarf es einer Wendung der Aufmerksamkeit hin zu den politischen Prozessen, den Institutionen und Regimen der Migrationskontrolle und zu ihrer Wissensproduktion.

2. Stellen Forschungen zur Sicht der solchen Regimen Unterworfenen auf Recht und Exekutive eine wesentliche Herausforderung dar, um blinde Flecken administrativen Wissens auszufüllen.

Das bedeutet zum einen, Migrationskontrolle als relevanten Entstehungskontext von Migrationswissen zu analysieren. Zum anderen impliziert es, das migrationspo-

\footnotetext{
1 So legten im Jahr 2004120 Regierungen in der International Agenda on Migration Management fest, dass es mehr ,,aktuelle(r), akkurate(r) und vergleichbare(r) Daten und weitere(r) Forschung zu allen Aspekten von Migration“ bedürfe, um eine ,,effektive“ Politik“ betreiben zu können (siehe IAMM 2005, S. 25, zit. n. Georgi und Wagner 2009, S. 306). Gefördert wird die Verzahnung von Wissenschaft und Politik zudem durch den gesteigerten Wert, den nationale und europäische Förderprogramme im Bereich der Migrationsforschung in den letzten Dekaden auf die Policy-Relevanz bei von ihnen finanzierten Projekten legen. Vor dem Hintergrund der gegenwärtigen ökonomischen Restrukturierung der Wissenschaft führt dies dazu, dass akademische ForscherInnen ihre Forschungsanträge vermehrt entlang hegemonialer Problemdefinitionen ausrichten und so staatlich-regulative Rationalitäten übernehmen (Dimitrova et al. u. a. 2009).
} 
litisch regierte Individuum nicht nur als Objekt der Kontrolle zu konzeptualisieren, sondern es vielmehr als aktives Element in einem komplexen Kontext wahrzunehmen, das seinerseits rechtliche Prozesse in Gang zu setzen vermag. Unter Heranziehung eines weiten, über die rechtswissenschaftliche Definition hinausgehenden Verständnisses von Recht - hier lehnen wir uns an Eugen Ehrlichs ,lebendes Recht““ an (1989) - bleibt empirisch zu untersuchen, ob überhaupt, und wenn ja, wie Recht und Rechtsinstitutionen für Individuen als Orientierungspunkte des Handelns dienen und auf welche Weise sie soziales Miteinander strukturieren. Auf beides sei in der Folge näher eingegangen, wenn auch in diesem Beitrag auf Letzteres der Schwerpunkt gelegt wird.

\section{Zur Notwendigkeit der Untersuchung von Migrationskontrollpraktiken}

Migration, das haben wir eingangs herausgestellt, lässt sich nicht ohne die Reflexion über legislative, judikative und exekutive Praktiken ihrer Regulierung untersuchen. Dies wird von der umfangreichen polizeilichen und kriminologischen Migrationsforschung hingegen vernachlässigt. Deshalb ist mit dem ersten Forschungsdesiderat nicht weniger als eine Perspektivenumkehr angesprochen, um Migration als administrative Kategorie in den Blick zu nehmen. Eine solche Forschung macht es sich zur Aufgabe, Fokussierungen, blinde Flecken und die Verwendung und Weiterentwicklung von Steuerungsinstrumenten zu untersuchen sowie die Generierung von Wissen aus der Kontrollpraxis kritisch zu reflektieren. Die zentrale Frage lautet: In welchem Zusammenhang stehen Rechts- und polizeiliche Praxen mit der ,(Il-) Legalität“ von Migration und der „Kriminalitätsbelastung“ von MigrantInnen?

Damit steht das hier zu skizzierende Forschungsdesiderat in direkter Tradition jenes Strangs einer kritischen Kriminologie, der sich weniger für „Kriminalität“ oder „Kriminelle“ interessiert, sondern stattdessen für jene kriminaljustiziellen Institutionen, die bestimmte Handlungen oder Personen(gruppen) so definieren (z. B. Sack 1968). Derartige kriminalsoziologische Arbeiten haben dazu beigetragen aufzuzeigen, wie sich über die Benennung des (strafrechtlich) Abweichenden das „Normale“ herzustellen pflegt. Ähnlich haben Arbeiten aus der Migrationsforschung beschrieben, wie die Markierung von Migration als dem Anderen bzw. Fremden zur Konstitution von Aufnahmegesellschaften beiträgt (z. B. Bielefeld 1998). Aktuelle Entwicklungen wie der Zusammenhang zwischen der zunehmenden Stratifizierung von Mobilität und Migration und der Konstruktion von Migration als Sicherheitsproblem lassen die Kombination beider Forschungstraditionen notwendig werden.

Denn in den letzten Dekaden lässt sich beobachten, wie ein abgestuftes System von Zugangsberechtigungen entlang der ökonomischen Verwertbarkeit von MigrantInnen entsteht, welches einerseits gespreizter, andererseits differenzierter ist als je zuvor und auch nicht mehr allein an das Staatsbürgerschaftskriterium geknüpft ist. Damit in Zusammenhang bringen lässt sich die Abwendung von einer Politik der Abschottung hin zum Management von Migration (z. B. Cuttita 2010). Der zugehörige Verwaltungs- und Rechtsapparat ist in diesem Zusammenhang enorm angewachsen und durchwuchert, d. h. er beteiligt oder verpflichtet auch andere Institutio- 
nen und Akteure (beispielsweise der privaten Transportwirtschaft). Methoden und Technologien zur Kontrolle von Zugangsberechtigungen, der Legalität von Reisen, Aufenthalten, Lebens- und Erwerbsweisen, sind verfeinert und zunehmend stärker verwoben mit Alltagsroutinen. Zwischen neuartigen Privilegierungen von „supracitizens“ (,bona fide travellers“, ,Globalmanagern“), ihrer Befreiung von Einschränkungen durch nationale Instanzen und der Zurücksetzung Anderer auf das ,nackte Leben“ (Agamben) - d. h. auf ein Leben ohne jeglichen Anspruch auf Bürgerrechte, an welchem Ort immer -, besteht ein innerer Zusammenhang. Es sind dieselben Technologien der Bewegungs- und Grenzkontrollen, die das eine wie das andere erleichtern und realisieren lassen. Datenvernetzung und Automation beschleunigen das Vorankommen der einen (zur „multi-speed citizenship“) und schaffen zugleich mehr Aufmerksamkeitskapazität für ,Illegalität“ und Kriminalität, somit für die Eindämmung nicht berechtigter gesellschaftlicher Teilhabeversuche (Aas 2011).

Wir sehen: Recht und (fremdenrechtliche) Verwaltung bilden heute ein Ungleichstellungssystem von globalem Maßstab, in dem ,global citizens“ und „crimmigrants“ (Aas 2011) praktisch unterschieden, diskriminiert und auch erst hervorgebracht werden (womit nur die beiden Enden eines weiten Spektrums bezeichnet sind). Zentral ist, dass Migrationskontrollen im Zuge dessen mit Kriminalitätskontrollen verschmelzen und gemeinsam und auch symbolwirksam der Abgrenzung von unsicheren gesellschaftlichen Außen- und (zumindest noch relativ) sicheren Innenräumen (Bigo 2001) dienen, sowie innerhalb letzterer zwischen Vollbürgern und Bürgern unter Vorbehalt, auf Zeit oder Bewährung (,sub-citizens“).

Die hier skizzierte sich ausbreitende Umdeutung unautorisierter grenzüberschreitender Mobilität zu einem kriminellen Tatbestand verwandelt eine globale immobile und entrechtete in eine illegale, kriminelle und gefährliche ,underclass“ (Pickerung und Weber 2006). Kriminalisierung betrifft auch die unberechtigte Inanspruchnahme sozialer Teilhaberechte durch Zugewanderte, wiewohl Missbrauchsmöglichkeiten erst durch zunehmend stratifizierte Rechte an sozialen Gütern und Leistungen geschaffen werden (Mohr 2005). Diese rechtlichen Entwicklungen werden durch „cross-border-crime“ oder „Sozialbetrug“ begründet und vorangetrieben, zugleich bringen sie diese Kriminalität wie auch die ausufernde polizeiliche Migrationskontrolle erst hervor. Anstatt sich in erster Linie auf Sicherheitsrisiken bzw. Kriminalitätsdispositionen bei MigrantInnen zu konzentrieren, ginge es somit darum, die (rechtliche, einschließlich der strafrechtlichen) Stratifizierung von Migration, deren Hintergründe, Methoden und Dynamiken, eingehender zu untersuchen.

In Bezug auf die hier skizzierten aktuellen Entwicklungen stehen Forschungen noch am Anfang. Zwar wurden derartige Zusammenhänge vor allem in der letzten Dekade von inter- bzw. post-disziplinären Forschungsfeldern wie der Kriminal(rechts-)soziologie, den surveillance und security studies und später auch den migration studies zum Gegenstand von Untersuchungen gemacht. ${ }^{2}$ Innerhalb der Migrationsforschung haben derartige Perspektiven auf Migrations-Kontrollregime

\footnotetext{
${ }^{2}$ Die diskursive und operative Verknüpfung von Migration mit „organisierter Kriminalität“ seit den 1980er-Jahren und dem ,internationalen Terrorismus“ seit Anfang des neuen Jahrtausends (vgl. Gutiérrez Rodríguez 2005, S. 73) hat hier vor allem die vielen Facetten einer „Versicherheitlichung“ (Buzan et al. 1998) von Migration in den Vordergrund treten lassen: Sei es im Rahmen einer „Politik der Menschenrechte“, der Verteidigung territorialstaatlicher Souveränitäten bzw. transnationaler Regime und ihrer
} 
jedoch insgesamt immer noch eine randständige Position. Aufgrund der positiven Entwicklungen, was dieses erste Desiderat kritischer kriminal(rechts)soziologischer Forschung betrifft, nämlich die Migrations- und Kriminalitätskontrollstrategien in ihrer Selbstverständlichkeit zu reflektieren und auf ihre (Wissens-)Effekte hin zu befragen, wollen wir es hier bei der groben Skizze belassen. Der vorliegende Beitrag widmet sich in den beiden nachfolgenden Abschnitten vor allem dem zweiten, wesentlich weniger beachteten Desiderat - der vertieften Auseinandersetzung mit den Erfahrungen von Unsicherheit, Konflikten, prekären Rechtspositionen und mit dem Umgang mit (Kriminal-)Rechtsressourcen durch MigrantInnen selbst und schließlich mit der Bedeutung dieser Erfahrungen und Praktiken für die Kriminalitäts- und Rechtswirklichkeit.

\subsection{Zur Notwendigkeit der Untersuchung subjektiver Perspektiven}

Eine von der so umfangreichen wie selektiven polizeilichen Datensammlung und ihren Routinen unabhängige und diese ergänzende und kontrastierende „kriminologische Migrationsforschung" existiert in Österreich bislang nicht, und auch in anderen Ländern sind solche Untersuchungen nur begrenzt vorhanden. Der nachfolgende Abschnitt bilanziert zunächst Versuche innerhalb der (internationalen) Kriminologie, Wissen über MigrantInnen unabhängig von den Polizeibehörden, von deren Gefährdungsannahmen und selektiver Kontrollpraxis zu gewinnen und zu vermehren. Er zeigt jedoch auch die Ergänzungsbedürftigkeit dieser Versuche, solange die Kriminologie bei ihren Dunkelfelderhebungen und Viktimisierungssurveys in den Kategorien des Strafrechts und seiner AnwenderInnen befangen bleibt. In Konsequenz dessen empfiehlt der dritte Abschnitt einen verallgemeinerten rechtsanthropologischen Blickwinkel, BürgerInnen insgesamt, so auch MigrantInnen, als ExpertInnen ihrer Lebenswelt, als InterpretInnen und (Ko-)ProduzentInnen ihres Rechts und ihrer eigenen Sicherheit zu beachten. Er fokussiert nicht länger auf „Kriminalität“, sondern auf die Abwehr oder Verarbeitung von Unsicherheit, Diskriminierung und Anspruchsverletzung durch Betroffene in unterschiedlichen Konstellationen und mit ihren jeweiligen sozialen Ressourcen, darunter den Mitteln des Rechts (der Verrechtlichung), darunter wiederum des Strafrechts (der Kriminalisierung). Jenseits etablierter Institutionen praktiziertes Konfliktregeln und Recht scheinen gerade im Bereich rechtlich unterprivilegierter Gruppen unterbelichtet und von besonderem Interesse. Die von hoher Selbstbewertung bis -überschätzung ihrer Funktion geprägte Perspektive der Administration im Allgemeinen und der (Straf-)Rechtspflege im Besonderen führte bisher zu einer Vernachlässigung der Vorfelder des institutionellen Rechts- und Verwaltungsbereiches. Dies sollte von der Forschung nicht länger mitvollzogen werden, nicht zuletzt, um Recht, Rechtsschutz und -fürsorge im Allgemeinen in ihrer emanzipatorischen Funktion weiterzuentwickeln (Steinert 2010).

\footnotetext{
Wohlfahrtsordnungen - stets konnte die Umcodierung von Migration und MigrantInnen in ein Sicherheitsproblem beobachtet werden (um nur einige zu nennen: Huysmans 2000; Fuchs und Kretschmann 2007; Janicki und Böwing 2010; Kasparek 2008).
} 


\subsection{Der Zugriff der Kriminologie und seine blinden Flecken}

Wer sich sozialwissenschaftlich mit dem Zusammenhang von Migration und Kriminalität beschäftigt, begibt sich auf ein „politisches und ideologisches Minenfeld“ (Eisner 1998, S. 11). So gibt es in nahezu allen westlichen Ländern Gruppen von Menschen fremder Ethnizität, die ein im Vergleich mit der autochthonen Bevölkerung erhöhtes Risiko aufweisen, angezeigt, verurteilt oder eingesperrt zu werden. Diese Überrepräsentation im „Hellfeld“ der offiziellen Kriminalstatistiken wird einerseits immer wieder herangezogen, um xenophobe Argumentationen und Stereotype zu bekräftigen. Andererseits ist genau diese - vermeintlich oder tatsächlich höhere Kriminalitätsbelastung einzelner ausländischer Bevölkerungsteile in den Augen kritisch-engagierter Stimmen nichts anderes als das Ergebnis rassistischer oder fremdenfeindlicher Praktiken der Strafverfolgungsorgane (vgl. Tonry 1997; Phillips und Bowling 2012). Die Heftigkeit der Vorwürfe, die im Diskurs um Kriminalität und Zuwanderung zumindest implizit immer auch mitverhandelt werden, zeigt an, dass die Materie alles andere als harmlos ist. Jenseits kulturalistischer Zuschreibungen geht es dabei stets um die durch Machtungleichgewichte strukturierten Beziehungen zwischen „Etablierten“ und „Außenseitern“, in denen erstere dazu neigen, letztere als „unbändige Übertreter von Gesetzen und Normen“ (Elias und Scotson 1990, S. 22) wahrzunehmen.

So hat sich die Kriminologie auch schon relativ früh mit Migrationsphänomenen beschäftigt. Klassische theoretische Konzepte der US-amerikanischen Kriminalsoziologie wie Anomie (vgl. Merton 1938), Kulturkonflikt (vgl. Sellin 1938), oder die sozialräumlichen Analysen der Chicago School (vgl. Shaw und McKay 1942) wurden maßgeblich durch Auseinandersetzungen zwischen Vertretern unterschiedlich privilegierter Gruppen inspiriert, die zu verschiedenen Zeitpunkten nach Nordamerika eingewandert waren. Das Hauptaugenmerk galt schon damals den jungen Männern der „Zweiten Generation“ von MigrantInnen, die im Gegensatz zu ihren meist besonders disziplinierten, bescheidenen und angepassten Eltern unangenehm auffielen. Ohne die Zustände ökonomischen Elends oder politischer Unterdrückung, denen die „erste Generation“ entronnen war, selbst kennengelernt zu haben, orientierten sie sich an den gesellschaftlich dominanten Maßstäben materiellen Erfolgs, waren aber gleichzeitig sozialstrukturell bedingt mit sehr eingeschränkten Möglichkeiten konfrontiert, diese allgemein wertgeschätzten kulturellen Ziele auch zu erreichen.

\subsubsection{Amtliche Daten und Wissenslïcken}

Ähnliche Wahrnehmungen, die allerdings nicht immer theoretisch reflektiert wurden, prägten auch die deutschsprachige kriminologische Debatte, die - wie in vielen anderen europäischen Ländern - im Gefolge der ersten Arbeitsmigrationswellen ab Ende der 1970er-Jahre einsetzte (vgl. Albrecht und Pfeiffer 1979). Die - gelegentlich als „,tickende soziale Zeitbombe“ (vgl. Schwind 1983) dramatisierte Beobachtung einer erhöhten Kriminalitätsbelastung von Gastarbeiternachkommen, die sich bis in die 1990er-Jahre hinein ganz überwiegend auf amtliche Daten stützte, blieb aber nicht ohne Widerspruch: Sie sei, wie kritische BeobachterInnen schon bald anmerkten, ein bloßes „Artefakt der Kriminalstatistik“ (Geißler und Marißen 1990). Abgesehen 
davon, dass die polizeilichen und gerichtlichen Datensammlungen ausschließlich das - im Hinblick auf Migrations- und Kriminalitätsphänomene nur bedingt relevante - Merkmal der Staatsangehörigkeit tatverdächtiger oder verurteilter Personen erfassen, gehört es heute unzweifelhaft zum gesicherten kriminologischen Wissen, dass ein allenfalls kriminalstatistisch nachweisbarer erhöhter Fremdenanteil nichts über eine erhöhte Kriminalitätsbelastung der ausländischen Wohnbevölkerung auszusagen vermag (vgl. Pfeiffer et al. 2005, S. 17 ff.). Da die Kriminalstatistiken auch Straftaten mobiler Populationen von Menschen mit fremder Staatsangehörigkeit erfassen, die über gar keinen ordentlichen Wohnsitz im Inland verfügen (Touristen, Durchreisende, Austauschstudierende, irregulär Zugewanderte etc.), ist es grob verzerrend, die gesamte Ausländerkriminalität auf die bevölkerungsstatistisch erfasste Ausländerwohnpopulation zu beziehen. Hinzu kommt, dass bestimmte Straftaten des Fremdenrechts begreiflicherweise fast ausschließlich von Personen begangen werden, die nicht dem Staat angehören, in den sie einwandern. Schließlich ist die demographische und sozioökonomische Struktur der Ausländerpopulation nicht mit der inländischen Wohnbevölkerung vergleichbar: Zugewanderte sind in der Regel ,jünger, überproportional männlich und vermehrt am unteren oder oberen Ende der sozialen Statusskala angesiedelt“ (Pilgram et al. 2012, S. 7). Die bisherigen „Periodischen Sicherheitsberichte" der deutschen Bundesregierung (BMI/BMJ 2001, 2006) verzichten aus diesen Gründen überhaupt auf Kriminalitätsbelastungsziffern für Nicht-Deutsche. Dieser politisch korrekte „Wille zum Nichtwissen“ mag nicht die schlechteste Lösung für die skizzierten Probleme im Umgang mit administrativen Kriminalitätsdaten sein. $\mathrm{Ob}$ er die politisch und wissenschaftlich interessierte kritische Öffentlichkeit auf Dauer zu überzeugen vermag, sei dahingestellt.

\subsubsection{Kriminologische Wissensproduktion}

Es liegt auf der Hand, dass kriminologische Befunde, die sich auf administrative Strafverfolgungsdaten stützen, von begrenzter Aussagekraft sind. Abgesehen davon, dass letztere nicht für wissenschaftliche Zwecke erhoben werden, sondern vor allem der Selbststeuerung und -darstellung der einschlägigen Organisationen dienen (vgl. Kreissl 2011), spiegeln sie als Anzeigen- und Tätigkeitsstatistiken nichts anderes als „Bilder der gesellschaftlich stattfindenden Kriminalisierungen“ (Pilgram 1980, S. 37) wider. $^{3}$ Über die Verbreitung kriminalisierbarer Handlungen, die im „Dunkelfeld" der nicht angezeigten oder entdeckten Straftaten verbleiben, können amtliche Kriminalstatistiken nichts aussagen. Jenseits der Staatsangehörigkeit enthalten sie auch keine Hinweise auf Migrationserfahrungen verdächtiger Personen (oder ihrer Eltern und Familien). Aus diesen Gründen hat die Kriminologie versucht, einen eigenen empirischen Zugang zum ,kriminellen Geschehen“ und seinem sozialen Kontext zu finden: mittels aufwändiger Umfragestudien, in denen neben Viktimisierungsund Tätererfahrungen auch sozioökonomische Merkmale und Einstellungsdimen-

\footnotetext{
3 Nach dem Stand der Forschung zu selektiv unterschiedlich ausfallenden Kriminalisierungsprozessen im Justizsystem ist es nicht von der Hand zu weisen, dass ausländische Tatverdächtige der Tendenz nach strafrechtlich schärfer verfolgt werden als Einheimische (vgl. Pfeiffer et al. 2005; BMI/BMJ 2006: 426; Pilgram et al. 2012, S. 24 ff.).
} 
sionen erhoben werden. In Deutschland werden solche Forschungsprojekte seit den 1990er-Jahren regelmäßig als Befragungen Jugendlicher in Schulen durchgeführt. Obwohl die Ergebnisse aufgrund unterschiedlicher Zielpopulationen, Stichprobenzusammensetzungen, Erhebungsinstrumente und Auswertungsmethoden weder völlig vergleichbar noch einheitlich sind, lassen sich etwas vereinfachend doch einige bemerkenswerte Übereinstimmungen und Tendenzen benennen (vgl. Walburg 2007, S. 245 ff.; Enzmann 2010, S. 63):

- Erstens zeigen sich im Hinblick auf die „Gesamtdelinquenzbelastung“, die stark durch jugendtypische Bagatelldelikte geprägt ist, kaum Unterschiede zwischen Jugendlichen mit und ohne Migrationshintergrund.

- Dasselbe trifft zweitens auf die meisten Formen von Eigentumsdelinquenz zu, bei der manche Gruppen migrantischer Jugendlicher sogar weniger Delikte berichten als die Befragten ohne Migrationshintergrund.

- Drittens geben Jugendliche mit Migrationshintergrund dagegen öfter als ihre ,einheimischen“ Altersgenossen an, Gewaltdelikte begangen zu haben. Zumindest für schwerwiegendere Verhaltensweisen verschwindet dieser Effekt aber oder wird wenigstens deutlich schwächer, wenn die - für migrantische Jugendliche in aller Regel schlechteren - sozioökonomischen Lebensbedingungen statistisch kontrolliert werden. Allenfalls zeigt sich in multivariaten Modellen eine Neigung männlicher Jugendlicher aus der „,zweiten Generation“, verstärkt das Begehen (überwiegend harmloser) Gewaltdelikte zu berichten, die nicht direkt auf die soziale Lebenslage zurückgeführt werden kann. Erklärbar ist dieses Kokettieren mit Gewalt durch gemessene Einstellungsdimensionen, die eine Akzeptanz ,gewaltlegitimierender Männlichkeitsnormen“ anzeigen. Solche Orientierungen können indes nicht einfach kulturalistisch gedeutet werden, sondern dürften ihrerseits eher als Anpassung an soziale Benachteiligungen und Marginalisierungen zu verstehen sein (vgl. Enzmann et al. 2004).

- Viertens hängt schließlich bei Konflikten zwischen Jugendlichen, die als Gewalterfahrung erlebt und berichtet werden, die Anzeigebereitschaft ganz wesentlich von der ethnischen Täter-Opfer-Konstellation ab: Jugendliche ohne Migrationshintergrund weisen ein weitaus geringeres Risiko auf, von Opfern (egal welcher Herkunft) angezeigt zu werden, als solche mit Migrationshintergrund (Mansel und Albrecht 2003; Köllisch 2009; Baier et al. 2009). Migrantische Jugendliche sind also bei Konflikten mit Gleichaltrigen einem größeren Risiko selektiver Kriminalisierung ausgesetzt, was (auch vor dem Hintergrund allenfalls erhöhter Anteile an Tatverdächtigen in der polizeilichen Kriminalstatistik) ein durchaus brisanter Befund ist - ein Befund, der aber keine Auskunft darüber gibt, warum die Mehrheit der Auseinandersetzungen dennoch ohne das Einschalten formeller Kontrollinstanzen geschlichtet werden kann und warum sich MigrantInnen bei Konflikten mit „einheimischen“ Jugendlichen seltener an die Polizei wenden.

Für Österreich muss der Stand des kriminologischen Wissens im Hinblick auf Migration im Vergleich mit anderen Ländern (z. B. Deutschland oder Großbritannien) als sehr eingeschränkt bezeichnet werden, was nicht nur mit der geringen Größe des Landes, sondern auch mit dem Fehlen einer nennenswerten Forschungstradition zu tun haben dürfte. Bis auf wenige Ausnahmen (Pilgram 2011; Pilgram et al. 2012) 
gibt es kaum aktuelle Studien, in denen amtliche Kriminalstatistiken systematisch, zusammenschauend und kritisch auf die Frage hin ausgewertet werden, welche validen Aussagen über die Ausländerpopulation sie überhaupt erlauben. Unmöglich sind solche Aussagen jedenfalls dann, wenn - wie etwa bei Reindl-Krauskopf und Grafl (2009) - nur absolute Zahlen oder Anteile fremder Tatverdächtiger aus der polizeilichen Kriminalstatistik berichtet werden.

Dunkelfeldstudien fehlen in Österreich ebenfalls nahezu gänzlich. Die bislang einzige Ausnahme war die zweite Erhebungswelle der ,International Self-Report Delinquency Study“ (ISRD 2), in deren Rahmen 2005/06 rund 3000 österreichische Jugendliche der 7. bis 9. Schulstufe in Wien, Graz und fünf weiteren vorab ausgewählten Mittel- und Kleinstädten befragt wurden (Stummvoll et al. 2010). Dieses „City-Sampling“" war durch das internationale Studiendesign vorgegeben. Die Daten dieser Erhebung, die auch Aussagen über den Zusammenhang von Migrationshintergrund und selbst berichteter Delinquenz erlauben (vgl. auch Fuchs und Krucsay 2011), wurden eigens für diesen Beitrag entsprechend ausgewertet. Die Ergebnisse sind in Tab. 1 dargestellt. Dort finden sich - jeweils nach Migrationshintergrund (gesamt sowie „erste“ und „Zweite“ Generation) aufgeschlüsselte - Angaben für den Gruppenanteil, den Anteil der Jugendlichen, der eine höhere Schule besucht, sowie die Anteile der Befragten, die angeben, ein bestimmtes Delikt in ihrem Leben schon einmal begangen zu haben.

Dargestellt sind die - durch entsprechende Fragebogenitems definierten - Delikte „Gruppenschlägerei“, „,Körperverletzung“, „Sachbeschädigung“ und „Ladendiebstahl" sowie der Anteil derer, die zumindest irgendeine der im Rahmen der Studie abgefragten verbotenen Verhaltensweisen (,Delinquenz gesamt“) berichten. Zusätzlich sind Odds Ratio-Werte angegeben, die den Faktor darstellen, um den sich die Chance der Jugendlichen mit Migrationshintergrund gegenüber der Referenzgruppe der Jugendlichen ohne Migrationshintergrund (beide Eltern in Österreich geboren) erhöht oder verringert, das Begehen eines bestimmtes Deliktes zu berichten. Diese Werte können mittels logistischer Regressionen für das Schulniveau kontrolliert werden, das - um die Auswertung bewusst einfach zu halten - als binäre Variable (Besuch einer höheren Schule: ja oder nein) gefasst wurde. Diese multivariate Kontrolle trägt dem Umstand Rechnung, dass Jugendliche mit Migrationshintergrund deutlich seltener höhere Schulen besuchen, was als Indikator für einen niedrigeren sozioökonomischen Status und eine geringere Ausstattung mit kulturellem Kapital (vgl. Bourdieu 1992) angesehen werden kann. Im Lichte des konventionellen theoretischen und empirischen Wissens der Kriminologie sollte dies die Wahrscheinlichkeit delinquenter Verhaltensweisen erhöhen.

Die Ergebnisse bestätigen recht eindrucksvoll die oben skizzierten Trends der deutschen Dunkelfeldforschung: Bereits ohne Kontrolle des Schulniveaus zeigen sich keine großen Unterschiede in der „Delinquenzbelastung“ zwischen Jugendlichen mit und ohne Migrationshintergrund. In der Gesamtbetrachtung sind migrantische Befragte sogar weniger belastet, wenn ihr niedrigerer sozioökonomischer Status berücksichtigt wird. Das signifikant häufigere Berichten von Gewaltdelikten bleibt nach multivariater Überprüfung nur für das vergleichsweise harmlose Delikt der „Gruppenschlägerei“ erhalten und das auch nur bei Jugendlichen der „Zweiten Generation“. Jugendliche, die selbst im Ausland geboren sind, geben hingegen sig- 
Tab. 1 Selbstberichtete Delinquenz und Migrationshintergrund im österreichischen Sample der ISRD 2Studie

\begin{tabular}{|c|c|c|c|c|c|}
\hline & \multirow{2}{*}{$\begin{array}{l}\text { Kein } \\
\text { Migrati- } \\
\text { onshin- } \\
\text { tergrund }\end{array}$} & \multicolumn{3}{|c|}{ Migrationshintergrund } & \multirow{2}{*}{$\begin{array}{l}\text { Gesamt } \\
\text { (gültiges } N \text { ) }\end{array}$} \\
\hline & & Gesamt & $\begin{array}{l}\text { Mindestens } \\
\text { ein Elternteil } \\
\text { im Ausland } \\
\text { geboren }\end{array}$ & $\begin{array}{l}\text { Selbst im } \\
\text { Ausland } \\
\text { geboren }\end{array}$ & \\
\hline Anteil (\%) & 63,9 & 36,1 & 20,8 & 15,3 & $100,0(2908)$ \\
\hline $\begin{array}{l}\text { Anteil in höherer Schule } \\
(\%)\end{array}$ & 65,2 & 38,2 & 43,6 & 30,9 & $55,5(2908)$ \\
\hline \multicolumn{6}{|l|}{ „Gesamtdelinquenz“ } \\
\hline Lebenszeitprävalenz (\%) & 41,7 & 40,7 & 45,9 & 33,6 & $41,3(2823)$ \\
\hline OR & - & 0,96 & $1,18^{*}$ & $0,71^{* * *}$ & - \\
\hline $\begin{array}{l}\text { OR nach Kontrolle } \\
\text { „Gruppenschlägerei“ }\end{array}$ & - & $0,85^{* *}$ & 1,07 & $0,60^{* * * *}$ & - \\
\hline Lebenszeitprävalenz (\%) & 18,7 & 24,0 & 28,8 & 17,5 & $20,7(2895)$ \\
\hline OR & - & $1,37^{* * *}$ & $1,75^{* * * *}$ & 0,92 & - \\
\hline $\begin{array}{l}\text { OR nach Kontrolle } \\
\text { „Körperverletzung“ }\end{array}$ & - & $1,18^{*}$ & $1,55^{* * * *}$ & $0,75^{* *}$ & - \\
\hline Lebenszeitprävalenz (\%) & 3,7 & 4,7 & 5,8 & 3,2 & $4,0(2893)$ \\
\hline OR & - & 1,29 & $1,62^{* *}$ & 0,86 & - \\
\hline $\begin{array}{l}\text { OR nach Kontrolle } \\
\text { „Sachbeschädigung“ }\end{array}$ & - & 1,12 & 1,43 & 0,71 & - \\
\hline Lebenszeitprävalenz (\%) & 17,8 & 14,7 & 15,6 & 13,5 & $16,7(2899)$ \\
\hline OR & - & $0,80^{* *}$ & 0,85 & $0,72^{* *}$ & - \\
\hline $\begin{array}{l}\text { OR nach Kontrolle } \\
\text { „Ladendiebstahl“ }\end{array}$ & - & $0,74^{* * *}$ & $0,80^{*}$ & $0,66^{* * *}$ & - \\
\hline Lebenszeitprävalenz (\%) & 19,1 & 15,7 & 17,6 & 13,2 & $17,9(2893)$ \\
\hline OR & - & $0,79^{* *}$ & 0,90 & $0,64^{* * *}$ & - \\
\hline OR nach Kontrolle & - & $0,71^{* * *}$ & 0,82 & $0,56^{* * * *}$ & - \\
\hline
\end{tabular}

Werte für Odds Ratio (OR) vor und nach statistischer Kontrolle des Schulniveaus mittels logistischer Regression Referenzkategorie: beide Eltern in Österreich geboren: ${ }^{*} p<0,1 ;{ }^{* *} p<0,05 ;{ }^{* * *} p<0,01 ;{ }^{* * * *} p<0,001$

nifikant seltener an, dieses Delikt begangen zu haben. Für diese Gruppe zeigt sich generell eine deutlich niedrigere Chance, delinquentes Verhalten zu berichten. Von allen Befragten mit Migrationshintergrund werden schließlich die Eigentumsdelikte „Sachbeschädigung“ und „Ladendiebstahl“" seltener berichtet.

Derartig entlastende Befunde vermögen das „Minenfeld“ der Debatte um Migration und Kriminalität zweifellos zu entschärfen. Insofern ist es zu begrüßen, wenn solche Daten unabhängig von medialen Skandalisierungskonjunkturen regelmäßig erhoben werden. Trotzdem sollten auch die Grenzen und Gefahren dieser Art von Forschung im Auge behalten werden. So ist es durchaus fraglich, ob sich Jugendliche mit und ohne Migrationshintergrund nicht unterscheiden, was Frageverständnis, Antwortverhalten und Auskunftsbereitschaft betrifft. Erkenntnistheoretisch gesehen ist es außerdem nicht „Kriminalität“, die in Self-Report-Studien gemessen wird, sondern allenfalls die Bereitschaft, im Kontext der Befragungssituation das 
Begehen oder Erleiden potenziell kriminalisierbarer, durch Fragebogenitems standardisiert umschriebener Verhaltensweisen zu berichten. Aus diesem Grund sind die Delikte in Tab. 1 auch in Anführungszeichen gesetzt - schließlich handelt es sich trotz der zum Teil kriminalrechtlichen Begriffe gerade nicht um polizeilich ermittelte oder gar richterlich geprüfte Sachverhalte, die tatsächlich unter Tatbestände des Strafgesetzbuches subsumiert worden wären. Dieser epistemologische Unterschied, den es bei jeglicher Interpretation von Dunkelfelddaten zu berücksichtigen gilt, ist alles andere als trivial. In den Augen mancher KritikerInnen wäre es denn auch schlicht „unwissenschaftliche Spekulation“ (Kunz 2008, S. 64), das Reportverhalten der Befragten als Hinweis auf das „wahre Kriminalitätsgeschehen“ zu deuten. Dunkelfeldbefragungen zeichnen ein ganz bestimmtes Kriminalitätsbild, das nicht „echter“ als die amtlichen Statistiken ist und daher nicht mit „,der“ Wirklichkeit verwechselt werden sollte.

Solche erkenntniskritischen Einwände wiegen zwar nicht eben leicht, unterschätzen jedoch möglicherweise die Validität von Selbstauskünften der Untersuchungssubjekte (vgl. Naplava und Oberwittler 2002; Phillips und Bowling 2012). Problematischer als die methodologischen Schwächen, die Umfragestudien stets vorgehalten werden können, ist indessen - so das hier vertretene Argument - die unweigerliche Nähe der Fragestellungen der Forschung zu selbst berichteter Delinquenz und Viktimisierung zu administrativen Strafverfolgungsbedürfnissen. Wenn Dunkelfeldstudien darauf angewiesen sind, sich zum Einwerben von Forschungsgeldern als anwendungsnahe Hilfsmittel zur Prävention oder Eindämmung von Jugendkriminalität zu verkaufen, sind die damit verbundenen Kontroll- und Problematisierungsinteressen unübersehbar. Aber auch wenn das nicht der Fall ist, erscheinen die Forschungsfragen von eigentlich vorwissenschaftlichen, bürokratienahen Relevanzkriterien zumindest überlagert, insofern erwartet wird, dass ihre Ergebnisse zu einer Art objektivierenden Korrektur der amtlichen Anzeigen- und Verurteilungsstatistiken beizutragen haben. Mit der „kriminalstatistischen Funktionalisierung“ (Wetzels 1995, S. 3) der Befragten geht außerdem ein einseitiger Fokus auf Jugend- und Gewaltkriminalität einher: „Täterbefragungen“, die für die gesamte Bevölkerung repräsentativ sein sollen, sind praktisch fast nur bei schulpflichtigen Jugendlichen zu realisieren, deren abweichendes Verhalten im öffentlichen Diskurs - zumal in Verbindung mit Integrationsfragen - vor allem als „Gewalt“ in Erscheinung tritt. Der paradigmatische ,kriminelle Fremde“, dessen statistisch aggregierte Existenz auf dem Prüfstand steht, ist dann meist der körperlich aggressive junge Mann südlicher oder östlicher Herkunft. Auch wenn es die aufrichtigen persönlichen Motive der Forschenden sein mögen, den durch populistische Politiker und Boulevardmedien hochgehaltenen Mythos der besonderen Kriminalität der Zugewanderten zu entlarven, so besteht hier eine gewisse Gefahr, die schrillen thematischen Rahmungen, die man eigentlich versachlichen möchte, indirekt zu unterstützen. Widerlegungen dummer Vorurteile sind anfällig dafür, sich der impliziten Logik dessen zu fügen, was sie in bester Absicht in Frage stellen wollen (es ließen sich schließlich auch ganz andere Fragen stellen). Aus dem Blick droht dabei zu geraten, welche Praktiken ,lebenden Rechts“ (Ehrlich 1989) sich eigentlich jenseits formeller oder quasiformeller Kategorien von Kriminalität und Kriminalisierung abspielen. 
Lässt man die positivistische Hoffnung, durch Self-Report-Studien das Dunkelfeld exakt zu vermessen, leichten Herzens fahren, so ist - sofern man deren Ergebnisse nicht von vornherein ideologiekritisch abwerten will - eine reflexiv-pragmatische Haltung möglich, die innerhalb der kriminologischen scientific community mittlerweile auch weitgehend konsensfähig sein dürfte. So räumt etwa Wetzels (1995, S. 7), einer der Proponenten der deutschen Dunkelfeldforschung, durchaus selbstkritisch ein, dass Kriminalität als interpretatives und normatives Konstrukt ,nicht ohne weiteres auf der Ebene individueller Erfahrungen ,objektiv` zählbar“ ist. Dennoch können, wie auch Steinert (2001, S. 117) aus einem explizit kritisch-kriminologischen Standpunkt heraus betont hat, Self-Report-Studien insofern ,verdienstvoll“" sein, als sie Hinweise auf Kriminalisierungsprozesse geben, die zwischen „Einheimischen“ und „Fremden“ selektiv unterschiedlich ablaufen. Solche Befunde sollten dann aber durch weitere Forschungsbemühungen ergänzt werden, die die Logik des Zählens von Ereignissen hinter sich lassen.

\section{3 Über den alltäglichen Umgang mit Konflikterfahrungen und Rechtsressourcen}

Die Kriminalrechtspraxis ist nicht nur das, was professionelle RechtsanwenderInnen daraus machen, und Kriminalität ist nicht nur das, was jene dazu erklären. Kriminalrecht und Kriminalität sind vor allem dadurch bestimmt, wozu das Strafrecht (mit seinen Kategorien) und seine Organe im gesellschaftlichen Alltag für brauchbar und angemessen befunden werden. Von Relevanz für diese Kategorien ist mithin, welcher Umgang mit Kriminalnormen und welcher Gebrauch von Polizei und Gericht unterschiedlichen Gruppen als „normal“ erscheint, was an „Kriminalisierung“ als selbstverständlich und was im Gegenzug als inadäquat empfunden und vermieden wird - nicht zuletzt auch auf Seiten der MigrantInnenpopulation. Dabei ist das Recht im Alltag zum einen ein praktisches Instrument zur Durchsetzung von individuellen Zielen, zum anderen aber auch ein kommunikatives Mittel zur symbolischen Bekräftigung von Kultur, Institutionen und sozialen Machtstrukturen (Silbey 2005). Recht wird nur ausnahmsweise in formellen Verfahren, weitestgehend jedoch außerhalb des institutionellen Rechtssystems verhandelt. Die Kategorien Recht, Rechtmäßigkeit/Legalität spielen im gesellschaftlichen Alltag in der Deutung von Normalität und Abweichung, in der Konstruktion von Gesellschaft insgesamt eine wesentliche strukturierende und stabilisierende Rolle.

Der instrumentelle Gebrauch von Recht steht mit den unterschiedlich verfügbaren Ressourcen für eine Kriminalisierung oder für alternative Konfliktbearbeitung und Streitschlichtung im Zusammenhang. Dabei scheinen in den unteren sozialen Schichten unter den rechtlichen Ressourcen vor allem zivilrechtliche noch stärker verknappt als kriminalrechtliche (Merry 1990). Überproportionale „Kriminalitätsraten“ innerhalb unterprivilegierter Gruppen (migrantisch oder nicht) sind insofern nicht bloß als Ausdruck eines ungleichen, sondern vor allem eines gespaltenen Rechtszugangs zu betrachten. Die Bekräftigung der gesellschaftlichen und der Rechtsordnung in der Form des Strafrechtsgebrauchs bedeutet symbolisch die Subordination der AnzeigeerstatterIn sowie ihres Kollektivs unter staatliche und (Rechts-)Autorität - stärker noch als die Inanspruchnahme von (zivilem) Privatrecht. Die Verfolgung persönli- 
cher Interessen mittels Polizei und Strafjustiz ist daher nicht nur unter Umständen praktisch erschwert, sondern auch auf der symbolischen Ebene für Unterprivilegierte und Minderheiten ein prekäres und ambivalentes Unterfangen. Forschungsdesiderate bestehen daher nicht nur im Hinblick auf die faktische Strafrechtsanwendung, auf deren Möglichkeiten und Hürden, sondern auch im Hinblick auf die normative Rationalisierung der Anwendung oder auch Nichtanwendung von Strafrechtsressourcen.

\subsubsection{Eine Studie, die eine migrationssoziologische Replikation verdient}

Bereits in den 1980er-Jahren unternahm man an der J. W. Goethe-Universität Frankfurt in Zusammenarbeit mit dem Institut für Rechts- und Kriminalsoziologie in Wien den Versuch, den Gebrauch des Strafrechts und seiner Instanzen neben alternativen Möglichkeiten des Umgangs mit Konflikten in den Blick zu bekommen (vgl. Hanak et al. 1989). Anstatt von bereits vollzogenen Kriminalitätsdeutungen auszugehen und die InterviewpartnerInnen unter Verwendung von bestehenden rechtlichen und administrativen Kategorien zu befragen ${ }^{4}$, war es Ziel des Forschungsprojektes, sich über die gesamte Bandbreite an „Ärgernissen und Lebenskatastrophen“, die von den InterviewpartnerInnen durchlebt worden waren, formelle wie informelle Strategien des Konfliktmanagements beschreiben zu lassen. Der Rückgriff auf Polizei und Strafrecht wurde bewusst als ,Sonderfall“ im Umgang mit ,unerfreulichen Ereignissen“ und „Konflikten“ betrachtet, den es in andere Strategien der Befragten einzuordnen galt. Unter welchen situativen Voraussetzungen scheint sich die Hinwendung zu Exekutive und Strafjustiz aus Sicht der Betroffenen als geeignetes „Instrument" zu erweisen, und welchen Anforderungen werden eher andere rechtliche und öffentliche, aber auch unterschiedliche informelle Ressourcen gerecht?

Ein ähnlich umfassendes Interesse wurde jenen Fällen und Interviewausschnitten entgegengebracht, in denen es um den Umgang mit den Folgen eines schadensverursachenden Geschehnisses ging. Das Durchleben von „Ärgernissen und Lebenskatastrophen" sollte nicht als zwingend negative Erfahrung und unbedingt zu vermeidendes Erlebnis verstanden werden. „Verlierer-“ und „Siegergeschichten“ wurden gleichermaßen in Betracht gezogen, um die möglichen Auswirkungen unterschiedlicher Strategien des Konfliktmanagements und der Schadensbehebung auf die Beziehungen der Beteiligten zu ergründen. Besondere Aufmerksamkeit widerfuhr den zur Verfügung stehenden rechtlichen und außerrechtlichen Instrumentarien, um mit den Folgen ,zu-Recht“ zu kommen. Welche Lösungs- oder Umgangsmöglichkeiten von den Betroffenen als erstrebenswert betrachtet und welche hingegen kaum wahrgenommen wurden, erschien gerade auch aus einer kriminalpolitischen Perspektive interessant.

\footnotetext{
4 Insgesamt wurden in 234 Interviews 1138 „Konflikt-Geschichten“ (Hanak et al. 1989, S. 207) gesammelt. In den Interviews wurde gezielt nicht nach „Kriminalitätserfahrungen“ gefragt, sondern zu Erzählungen über erlebte „Konflikte“, „Auseinandersetzungen“, „Ärger“, „Schwierigkeiten“ aufgefordert, um ein möglichst umfassendes Forschungsinteresse zu bekunden. Die Ergebnisse der Datenanalyse sind für Ereignisse im städtischen Raum repräsentativ, nicht jedoch für Personen oder soziale Schichten.
} 
Eigentlicher Untersuchungsgegenstand waren somit Situationen und Prozesse und nicht Personen-/Gruppenmerkmale und Einstellungen (ebenda, S. 207). Trotzdem scheint es aus heutiger Perspektive bemerkenswert, dass zwar die sozioökonomische Statusposition in der Untersuchungspopulation auf Repräsentativität kontrolliert, dem Migrationshintergrund der Befragten vom Forschungsteam jedoch keine gesonderte Aufmerksamkeit entgegen-gebracht wurde. So wenig wie die Studie davon ausgeht, dass die Deutung von Unfällen, Konflikten oder Schadensfällen als „kriminell“, die praktische Initiation von Strafverfolgung und die formelle Inkriminierung auf der Hand liegen, sind die unter MigrantInnen herrschenden Regeln für den alltäglichen Umgang mit Kriminalnormen, mit Polizei und der Kriminaljustiz evident. Hinsichtlich dieser Regeln könnten sich MigrantInnen von anderen Gesellschaftsmitgliedern infolge ihres legalen Status und alternativer Vorstellungen von Fairness und davon, was Recht und Rechtsprechung dazu beitragen, unterscheiden.

Finden sich infolgedessen Hinweise auf ein gar nicht so universelles und einheitlich gehandhabtes Strafrecht, sondern vielmehr auf voneinander abweichende Vorstellungen von Strafrecht und auf divergente Regeln und Praktiken seiner Anwendung unter der autochthonen und der MigrantInnenpopulation? Werden die unterschiedlichen Anwendungsregeln bewusst als kulturspezifisch different und als Identitätsmerkmal und Mitteilung an die umgebende Gesellschaft wahrgenommen? Oder wird spezifisches Verhalten gegenüber Polizei und Justiz an den Tag gelegt, weil eine klassen- oder kulturspezifische Ungleichbehandlung erwartet wird; besteht also tatsächlich kein andersgelagertes „Rechtsbewusstsein“ auf Seiten der MigrantInnen?

Um eine Auseinandersetzung mit diesen Fragen zu ermöglichen, kann es nicht nur darum gehen, den Migrationshintergrund bei der Auswahl der InterviewpartnerInnen zu berücksichtigen. Auch der analytische Blick müsste für diese Fragestellung neu geschärft werden. Auf diese Weise ließe sich die Vielfalt an Beschränkungen und Übervorteilungen erfassen, denen MigrantInnen im alltäglichen Leben begegnen (können). Weiters könnten die vielfältigen Umgangsweisen einer AkteurInnengruppe analysiert werden, die in sich äußerst heterogen ist und keinesfalls essentialisiert werden sollte.

Um ein solches Forschungsdesiderat weiter zu konkretisieren, sind weitere methodische Reflexionen nötig. In jüngerer Zeit nimmt die Popularität narrativer und lebensbiographischer Interviews in der Migrationsforschung zu (vgl. z. B. Latcheva und Herzog-Punzenberger 2011) - eine Entwicklung, die sich auch für die Erörterung der hier besprochenen kriminologischen und rechtssoziologischen Fragestellungen als erkenntnisfördernd erweisen könnte. Abgesehen von den auf der Hand liegenden Erschwernissen, die bei solchen Methoden auf Grund der Abhängigkeit von Sprachkenntnissen zu erwarten sind, besteht die hier zentral fokussierte Problematik in der Präsentation von Analyseergebnissen unter relativer Vernachlässigung des Untersuchungsprozesses (z. B. Riemann 2003). Im Hinblick auf die konkrete Analyse des Datenmaterials bedeutet das, dass wissenschaftliche Nachvollziehbarkeit kaum gegeben ist. Zudem kann ein solcher Fokus die selbstreflexive Auseinandersetzung mit dem eigenen Tun der ForscherInnen allzu leicht in den Hintergrund rücken lassen. Um diesen Schwachpunkt aufzuzeigen und zu mindern, lässt sich Riemanns Methode anführen: Sie ließ unterschiedliche ForscherInnen mit unter- 
schiedlichem (Migrations-)Hintergrund dasselbe Transkript eines Interviews bearbeiten. Durch solch ein Vorgehen entsteht zumindest die Möglichkeit, ein höheres $\mathrm{Ma} ß$ an Intersubjektivität zu erzielen, wobei die Bedeutung von Selbstreflexivität und Perspektivenvielfalt gerade bei solchen Analysen besonders hervorzuheben ist, bei denen InterpretIn und Interviewte/r aus unterschiedlichen sozialen Kontexten kommen.

Um im Hinblick auf eine Replikationsstudie den Faden auch auf theoretischer Ebene noch etwas weiterzuspinnen, sei noch auf eine für die Untersuchung der Kriminalrechtspraxis und ihrer normativen und kulturellen Reflexion durch MigrantInnen wesentliche Unterscheidung hingewiesen: Potenziell kann sich eine jede Person als eine in ihren Rechten verletzte oder als eine das Recht verletzende vorfinden und entsprechend wechselhaft ins Verhältnis zum Strafrecht treten; im einen Fall in der Rolle als aktiv verfolgende, im anderen Fall als passiv verfolgte Person. Jedoch kann der Umgang mit der Opfer- wie auch mit der Täterrolle im Strafrecht ein sowohl passiv defensiver wie ein aktiver und offensiver sein. Der Handlungsspielraum zwischen einer Negation von Opferrollen, der misstrauischen Ablehnung des Opferschutzes im Rahmen des Strafrechts oder aber auch der pönalen Exploitation des Opferstatus ist groß. Er wird in unterschiedlichen sozialen Konstellationen und Situationen in verschiedener Weise ausgefüllt werden. Was für die Opferseite gilt, gilt auch für die TäterInnenrolle. Die Kritik am Recht und die Leugnung von Übertretungen, die „Normalisierung“, Erklärung und Rechtfertigung bzw. Übernahme von Verantwortung, aber auch eine „Hyperkonformität“ und Rechtsunterwerfung sind mögliche Antworten auf drohende Strafverfolgung.

\subsubsection{Wie viel „Recht“ und „Gericht“ erscheinen als normal?}

Über die konkreten Erfahrungen und Praktiken von MigrantInnen in der einen oder anderen Position hinaus, stellen sich weitere Fragen: Schätzen MigrantInnen Strafnormen und Strafrechtspraxis in der Aufnahmegesellschaft ihrer Situation und Kultur als gerecht ausgestaltet ein? Wird das Strafrecht in Aktion als fremd, bedrohlich oder als Chance wahrgenommen? Wird das Ausmaß der Kriminalität und Unsicherheit, das in der Gesellschaft herrscht, das von MigrantInnen verursacht wird oder von diesen erduldet werden muss, als beherrschbarer Normalzustand, als irritierend oder (und in welchen Aspekten) als Krise des Rechts und staatlicher Autorität gedeutet?

An die Frage, welche Konfliktregelung in verschiedenen Teilen der Gesellschaft mit und ohne staatliche Kriminalrechtsinstitutionen gepflogen wird, schließt sich eine allgemeinere an. Vereinfacht formuliert lautet sie: Wie viel „Recht“ und „Gericht“ erscheinen als normal? Damit etwa ist die Frage nach dem „Wie viel“ an „Verrechtlichung“, an rechtlicher Rahmung von Problemen und Konflikten angesprochen. Weiter interessiert, wie viel Handeln von formellen, öffentlich-rechtlichen Institutionen wie Polizei und Justiz aus der Perspektive unterschiedlicher Segmente der Gesellschaft überhaupt beansprucht wird. Inwieweit will man Konflikt- und Problemlösungen an Dritte im Allgemeinen und an Rechts(fürsorge)instanzen im Besonderen abgetreten sehen? Wie weit unterscheiden sich hier die kulturell geprägten normativen Ansprüche nicht nur von MigrantInnengruppen (Biografien, Generationen), sondern auch innerhalb der autochthonen Gesellschaft? Und wie weit sieht man 
diese Erwartungen de facto erfüllt bzw. erfüllbar, enttäuscht oder von Enttäuschung bedroht?

Welche normativen Maßstäbe werden für die ,(Nicht-)Veröffentlichung von Privatem" und für die Bezugnahme auf abstrakte rechtliche Regulative, etwa in familiären oder geschäftlichen Angelegenheiten, in Beziehungen innerhalb der eigenen sozialen, ethnischen oder religiösen Gruppe/Community oder aber in sozialen „Außenbeziehungen“ angelegt? Es handelt sich um normative Vorstellungen von Vertrauensschutz und Diskretion, von Autonomie und Ehre, aber auch von gebührender Rangordnung und unberechtigter Diskriminierung, von Statussicherung und Disziplinierungsbedarf, -recht und -gewalt. Wie wiederum werden diese Normen des Zusammenlebens und Rechtsgebrauchs sozial (informell) kontrolliert, und in welchen Situationen, von wem und mit welchen Konsequenzen werden sie übertreten?

Es ist bekannt, dass hierfür nicht nur der mehr oder weniger fremde kulturelle Hintergrund von MigrantInnen, sondern auch ihr legaler und sozialer Status in der Aufnahmegesellschaft ausschlaggebend sind. Der Rückgriff auf traditionelle Rechtsvorstellungen, die Bindung an Kollektive von Zuwandernden gleicher Herkunft und die außergerichtliche Streitregelung in lokalen informellen Einrichtungen kann in gleicher Weise kulturellem Gepäck wie den mangelnden Optionen aufenthaltsrechtlich illegaler oder ungesicherter Existenzen geschuldet sein. Die Stratifizierung innerhalb von Zuwanderergruppen, ungleiche Aufenthaltssicherheit und migrationsoder allgemeinrechtliche Kenntnis auf Seiten der MigrantInnen können traditionelle Abhängigkeitsverhältnisse in Zuwanderergemeinschaften und somit Segregationsverhältnisse stützen. Sie können die Entwicklung von Kontroll- und Rechtspraktiken in Anlehnung an die Rechtskultur der Herkunftsgesellschaft befördern, wobei die Zwangswirkung eines restriktiven Ausländerrechts fehlende traditionelle gemeinschaftliche Sanktionsmöglichkeiten substituiert (Kohlhagen 2006). Das Bewusstsein von „Illegalität“ oder migrantischer Prekarität seitens der Betroffenen wie der (legalen) MigrantInnenpopulation und der legitimierende, kompensierende, duldende oder exploitative Umgang damit sind wenig untersuchte Sachverhalte. Aufgrund von entsprechenden Erfahrungen stellt sich bei MigrantInnen vermutlich ein tendenziell distanziertes, flexibles und pragmatisches Verhältnis zu geltenden Rechtsnormen in der Zuwanderungsgesellschaft sowie ein selektiver Rekurs auf hergebrachte Sittennormen ein.

Ganz allgemein macht die Heterogenität heutiger Einwanderungsgesellschaften darauf aufmerksam, dass inzwischen wohl eher eine Koexistenz von Rechtsvorstellungen und „Rechtspflege“-Mechanismen, dass ein „Rechtspluralismus“ eher als ein einheitliches Recht für alle als die gesellschaftliche Normalität zu gelten hat (Pilgram u. a. 2012). Dies wiederum lässt die Frage akut werden, wie sich die Rechtsinstitutionen und die handelnden Personen auf eine solche Pluralität bzw. Pluralisierung einstellen, wieweit sie abweichendes Rechtsbewusstsein und Rechtsgebrauch von MigrantInnen wahrnehmen oder unterstellen, als legitim anerkennen und berücksichtigen, oder ob sie es problematisieren und durch eine besondere Rechtspraxis korrigierend beantworten.

Die Existenz von spezifischen Normen und Instanzen zu ihrer Kontrolle und Durchsetzung in ethnischen und religiösen Minderheiten wird unterschiedlich bewertet, noch bevor sie hinsichtlich Umfang und Auswirkung für die unterschiedli- 
chen Mitglieder der Minderheit (vor allem auch für Minderheiten oder Benachteiligte innerhalb derselben) untersucht ist. In Großbritannien sind minority legal orders seit längerem ein Thema, und es werden die Vorteile zur Kenntnis genommen, die sie für Minderheiten gegenüber den staatlichen Rechtsinstitutionen attraktiv machen. Man beschäftigt sich in weiterer Folge mit den möglichen und wünschenswerten Einflüssen des Mehrheitsrechts auf den Rechtsgebrauch der Minderheit und mit dessen Rückwirkungen auf das staatliche Rechtswesen (Malik 2012). Auf rechtspolitischer Ebene wird eine Diskussion über den staatlichen Umgang mit minoritären Rechtssystemen geführt. Die Antworten reichen von Prohibition, über Nichteinmischung, Anerkennungsregulative bis hin zu „Mainstreaming“ (der Hereinnahme von Elementen des Minderheitenrechts in das übergeordnete staatliche Rechtssystem). Eine empirische Beschäftigung mit Rechtsvorstellungen und -gebrauch in MigrantInnengruppen könnte einer derartigen Debatte Orientierungshilfen bieten und sie von Dogmatismen befreien.

Open access funding provided by University of Vienna.

Open Access Dieser Artikel wird unter der Creative Commons Namensnennung 4.0 International Lizenz (http://creativecommons.org/licenses/by/4.0/deed.de) veröffentlicht, welche die Nutzung, Vervielfältigung, Bearbeitung, Verbreitung und Wiedergabe in jeglichem Medium und Format erlaubt, sofern Sie den/die ursprünglichen Autor(en) und die Quelle ordnungsgemäß nennen, einen Link zur Creative Commons Lizenz beifügen und angeben, ob Änderungen vorgenommen wurden.

\section{Literatur}

Aas, Katja Franko. 2011. Crimmigrant ' bodies and bona fide travelers: surveillance, citizenship and global governance. Theoretical Criminology 15:331-346.

Albrecht, Peter-Alexis, und Christian Pfeiffer. 1979. Die Kriminalisierung junger Ausländer. Befunde und Reaktionen sozialer Kontrollinstanzen. München: Juventa.

Baier, Dirk, Christian Pfeiffer, Julia Simonson, und Susann Rabold. 2009. Jugendliche in Deutschland als Opfer und Täter von Gewalt. Erster Forschungsbericht zum gemeinsamen Forschungsprojekt des Bundesministeriums des Inneren und des KFN. Forschungsbericht Nr. 107. Hannover: Kriminologisches Forschungsinstitut Niedersachsen.

Bielefeld, Ulrich (Hrsg.). 1998. Das Eigene und das Fremde. Neuer Rassismus in der Alten Welt? Hamburg: Hamburger Edition.

Bigo, Didier. 2001. The Möbius ribbon of internal and external securities. In Identities, borders, orders. Rethinking international relations theory, Hrsg. Mathias Albert, David Jacobson, und David Lapid, 91-116. Minneapolis: Universität of Minnesota Press.

Bourdieu, Pierre. 1992. Die verborgenen Mechanismen der Macht. Hamburg: VSA.

Bundesministerium des Inneren, und Bundesministerium der Justiz. 2001. Erster Periodischer Sicherheitsbericht. Berlin: BMI, BMJ.

Bundesministerium des Inneren, und Bundesministerium der Justiz. 2006. Zweiter Periodischer Sicherheitsbericht. Berlin: BMI, BMJ.

Buzan, Barry, Ole Waever, und Jaap de Wilde. 1998. Security. A new framework for analysis. Boulder: Lynne Rienner.

Cuttitta, Paolo. 2010. Das europäische Grenzregime: Dynamiken und Wechselwirkungen. In Grenzregime. Diskurse, Praktiken, Institutionen in Europa, Hrsg. Sabine Hess, und Bernd Kasparek, 23-42. Berlin Hamburg: Assoziation A.

Dimitrova, Petja, et al. 2009. Wissen - Macht - UniStreik. Umkämpfte Wissensproduktion, (kritische) Migrationsforschung und das Recht auf Mobilität im Bologna-Prozess. malmoe 48:9.

Ehrlich, Eugen. 1989. Grundlegung der Soziologie des Rechts, 4. Aufl., Berlin: Duncker \& Humblot.

Eisner, Manuel. 1998. Konflikte und Integrationsprobleme. Neue Kriminalpolitik 4(98):11-13.

Elias, Norbert, und John L. Scotson. 1990. Etablierte und Außenseiter. Frankfurt am Main: Suhrkamp. 
Engle Sally, Merry. 1990. Getting justice and getting even: legal consciousness among working class americans. Chicago: University of Chicago Press.

Enzmann, Dirk. 2010. In Juvenile Delinquency in Europe and Beyond. Results of the Second International Self-Report Delinquency Study, Hrsg. Josine Junger-Tas et al., 47-64. Dordrecht Heidelberg London: Springer.

Enzmann, Dirk, Katrin Brettfeld, und Peter Wetzels. 2004. Männlichkeitsnormen und die Kultur der Ehre. Empirische Prüfung eines theoretischen Modells zur Erklärung erhöhter Delinquenzraten jugendlicher Migranten. In Soziologie der Kriminalität Sonderheft 43 der Kölner Zeitschrift für Soziologie und Sozialpsychologie., Hrsg. Dietrich Oberwittler, und Susanne Karstedt, 264-287. Wiesbaden: VS.

Fuchs, Walter, und Andrea Kretschmann. 2007. Legitimationstechniken, Sicherheitspraktiken: Von der Normalität des Staatsverbrechens. Eine erkenntnistheoretische Diskussion am Beispiel des Falles Murat Kurnaz. Kriminologisches Journal 39(4):260-277.

Fuchs, Walter, und Brita Krucsay. 2011. Zählen und Verstehen: Jugenddelinquenz, erfahrungswissenschaftlich betrachtet. In 6. Bericht zur Lage der Jugend in Österreich, Hrsg. Bundesministerium für Wirtschaft, Familie und Jugend, 353-375. Wien: Bundesministerium für Wirtschaft, Familie und Jugend.

Geißler, Rainer, und Norbert Marißen. 1990. Kriminalität und Kriminalisierung junger Ausländer. Die tickende soziale Zeitbombe - ein Artefakt der Kriminalstatistik. Kölner Zeitschrift für Soziologie und Sozialpsychologie 42:663-687.

Georgi, Fabian, und Fabian Wagner. 2009. Macht, Wissen, Kontrolle. Bedingungen kritischer Migrationsforschung. kulturrisse 1:24-28.

Gutiérrez Rodríguez, Encarnación. 2005. Das postkoloniale Europa dekonstruieren. Zu Prekarisierung, Migration und Arbeit in der EU. Widerspruch 48(5):71-84.

Hanak, Gerhard, Johannes Stehr, und Heinz Steinert. 1989. Ärgernisse und Lebenskatastrophen. Über den alltäglichen Umgang mit Kriminalität. Bielefeld: AJZ.

Hess, Sabine, und Bernd Kasparek. 2010. Einleitung. Perspektiven kritischer Migrations- und Grenzregimeforschung. In Grenzregime. Diskurse, Praktiken, Institutionen in Europa, Hrsg. Sabine Kasparek, und Bernd Kasparek, 7-22. Berlin Hamburg: Assoziation A.

Huysmans, Jef. 2000. The European Union and the securitization of migration. Journal of Common Market Studies 38(5):751-777.

Janicki, Jill Jana, und Thomas Böwing. 2010. Europäische Migrationskontrolle im Sahel. Das CIGEM in MALI. In Grenzregime. Diskurse, Praktiken, Institutionen in Europa, Hrsg. Sabine Hess, und Bernd Kasparek, 127-144. Berlin Hamburg: Assoziation A.

Kasparek, Bernd. 2008. Frontex oder die Krise der europäischen Migrationspolitik. Die Europäisierung der Migrationspolitik. diskus 1:32-35.

Kohlhagen, Dominik. 2006. „Illegale“ Migration und Rechtskultur - Beobachtung aus einer Feldforschung unter Afrikanern in Deutschland. Zeitschrift für Rechtssoziologie 27:239-250.

Köllisch, Tilman. 2009. Vom Dunkelfeld ins Hellfeld. Zur Theorie und Empirie selektiver Kriminalisierung Jugendlicher bei Körperverletzungsdelikten. Monatsschrift für Kriminologie und Strafrechtsreform 92:28-53.

Kreissl, Reinhard. 2011. Governing by numbers. In Uneindeutigkeit als Herausforderung - Risikokalkulation, Amtliche Statistik und die Modellierung des Sozialen, Hrsg. Daniel Fischer et al., 53-66. Neubiberg: Universität der Bundeswehr München.

Kunz, Karl-Ludwig. 2008. Die wissenschaftliche Zugänglichkeit von Kriminalität. Ein Beitrag zur Erkenntnistheorie der Sozialwissenschaften. Wiesbaden: Deutscher Universitätsverlag VS.

Latcheva, Rossalina, und Barbara Herzog-Punzenberger. 2011. Integration revisited. Österreichische Zeitschrift für Soziologie 36:3-27.

Malik, Maleiha. 2012. Minority legal orders in the UK. London: The British Academy.

Mansel, Jürgen, und Günter Albrecht. 2003. Die Ethnie des Täters als ein Prädiktor für das Anzeigeverhalten von Opfern und Zeugen. Die private Strafanzeige als Form der Konfliktregulierung. Soziale Welt 54:339-372.

Merton, Robert K. 1938. Social structure and anomie. American Sociological Review 3(5):672-682.

Mohr, Katrin. 2005. Stratifizierte Rechte und soziale Exklusion von Migranten im Wohlfahrtsstaat. Zeitschrift für Soziologie 34:383-398.

Naplava, Thomas, und Dietrich Oberwittler. 2002. Methodeneffekte bei der Messung selbstberichteter Delinquenz von männlichen Jugendlichen. Ein Vergleich zwischen schriftlicher Befragung in der Schule und mündlicher Befragung im Haushalt. Monatsschrift für Kriminologie und Strafrechtsreform $85: 401-423$. 
Pfeiffer, Christian, Matthias Kleinmann, Sven Petersen, und Tilmann Schott. 2005. Migration und Kriminalität. Ein Gutachten für den Zuwanderungsrat der Bundesregierung. Baden-Baden: Nomos.

Phillips, Coretta, und Ben Bowling. 2012. Ethnicities, Racism, Crime and Criminal Justice. In The Oxford Handbook of Criminology, Hrsg. Mike Maguire, Rod Morgan, und Robert Reiner, 370-397. Oxford: Oxford University Press.

Pickering, Sharon, und Leanne Weber. 2006. Borders, mobility and technologies of control. Dordrecht: Springer Netherlands.

Pilgram, Arno. 1980. Kriminalität in Österreich. Studien zur Soziologie der Kriminalitätsentwicklung. Wien: Verlag für Gesellschaftskritik.

Pilgram, Arno. 2011. Kriminalität und strafrechtliche Sozialkontrolle von MigrantInnen. In Grundzüge Migrationsmanagement, Bd. 1, Hrsg. Gudrun Biffl, und Nikolaus Dimmel. Vöslau: omnium.

Pilgram, Arno et al. (Hrsg.). 2012. Einheitliches Recht für die Vielfalt der Kulturen? Strafrecht und Kriminologie in Zeiten transkultureller Gesellschaften und transnationalen Rechts. Münster: LIT.

Pilgram, Arno, Walter Fuchs, und Norbert Leonhardmair. 2012. Welche Aussagen über die Migranten- und Ausländerpopulation in Wien erlaubt die Kriminalstatistik? Wien: Institut für Rechts- und Kriminalsoziologie.

Reindl-Krauskopf, Susanne, und Christian Grafl. 2009. Kriminalität nicht integrierter Ausländer - eine vielfältige Herausforderung für das Strafrecht. Verhandlungen des Siebzehnten Österreichischen Juristentages, Bd. IV/1. Wien: Manz.

Riemann, Gerhard. 2003. A joint project against the backdrop of a research tradition: an introduction to “doing biographical research". Forum Qualitative Sozialforschung 4(3), Art. 18.

Sack, Fritz. 1968. Neue Perspektiven in der Kriminologie. In Kriminalsoziologie, Hrsg. René König, 430-475. Frankfurt am Main: Akademische Verlagsgesellschaft.

Schwind, Hans-Dieter. 1983. Wie lösen wir die Ausländerfrage? Das Gastarbeiterproblem aus (kriminal-)politischer Sicht. Kriminalistik 37(6):303-306.

Sellin, Thorsten. 1938. Culture conflict and crime. New York: Social Science Research Council.

Shaw, Clifford R., und Henry D. McKay. 1942. Juvenile delinquency in urban areas. Chicago: The University of Chicago Press.

Silbey, Susan S. 2005. After legal consciousness. Annual Reviews Law and Society Science 1:328-368. http://web.mit.edu/ssilbey/www/pdf/after_legal.pdf.

Steinert, Heinz. 2001. Kulturindustrie und die Zivilisierung der Gewalt. In Gewaltkriminalität zwischen Mythos und Realität, Hrsg. Günter Albrecht, Otto Backes, und Wolfgang Kühnel, 101-122. Frankfurt am Main: Suhrkamp.

Steinert, Heinz. 2008. Die Widerständigkeit der Theorie. In Das Elend der Universitäten. Neoliberalisierung deutscher Hochschulpolitik, Hrsg. Jens Sambale, Volker Eick, und Heike Walk, 155-170. Münster: Westfälisches Dampfboot.

Steinert, Heinz. 2010. Gegen-Institutionen und Gegen-Wissen im Strafrecht: am Beispiel des Instituts für Rechts- und Kriminalsoziologie. juridikum 1:37-45.

Stummvoll, Günter P., Ingrid Kromer, und Isabella Hager. 2010. Austria. In Juvenile delinquency in Europe and beyond. Results of the second international self-report delinquency study, Hrsg. Josine JungerTas et al., 97-109. Dordrecht: Springer.

Tonry, Michael. 1997. Ethnicity, crime and immigration. In Ethnicity, crime and immigration. Comparative and cross-national perspectives, Hrsg. Michael Tonry, 1-29. Chicago: The University of Chicago Press.

Walburg, Christian. 2007. Migration und selbst berichtete Delinquenz. In Delinquenz im Jugendalter. Erkenntnisse einer Münsteraner Längsschnittstudie, Hrsg. Klaus Boers, und Jost Reinecke, 241-268. Münster: Waxmann.

Wetzels, Peter. 1995. Wider den naiven Realismus kriminologischer Opferforschung. Plädoyer für einen subjektiven, konstruktivistischen Opferbegriff. Forschungsbericht Nr. 45. Hannover: Kriminologisches Forschungsinstitut Niedersachsen.

Young, Jock. 1986. Failure of criminology: the need for a radical realism. In Confronting crime, Hrsg. Roger Matthews, und Jock Young, 4-30. London: SAGE.

Walter Fuchs ist wissenschaftlicher Mitarbeiter am Institut für Rechts- und Kriminalsoziologie. Er war von 2012 bis 2015 Stipendiat (APART) der Östereichischen Akademie der Wissenschaften, ist seit 2011 Lektor am Institut für Soziologie der Universität Wien und gibt das „Kriminologische Journal“ sowie die „Zeitschrift für Rechtssoziologie“ mit heraus; ausgewählte Forschungsschwerpunkte: Rechtssoziologie, abweichendes Verhalten und soziale Kontrolle, Erwachsenenvertretungsrecht. 
Katrin Kremmel studierte Kultur- und Sozialanthropologie in Wien, Barcelona und São Paulo, ist ehemalige wissenschaftliche Mitarbeiterin des Instituts für Rechts- und Kriminalsoziologie und arbeitet seit Juni 2016 als praedoc Universitätsassistentin am Institut für Kultur- und Sozialanthropologie der Universität Wien.

Andrea Kretschmann ist Soziologin und Kriminologin und derzeit als Forscherin am Centre Marc Bloch, An-Institut der Humboldt-Universität zu Berlin, angesiedelt. Sie ist Mitherausgeberin des „Kriminologischen Journals“ und Redakteurin des ,juridikum“. Ausgewählte Forschungsschwerpunkte: Rechts- und Kriminalsoziologie, Soziologie der Gewalt und des Konflikts, soziologische Theorie.

Arno Pilgram ist Soziologe, habilitiert an der J.W.-Goethe-Universität Frankfurt/Main und an der Universität Wien, ehemaliger Wissenschaftlicher Leiter des Instituts für Rechts- und Kriminalsoziologie, Mitherausgeber der „Schriften für Rechts- und Kriminalsoziologie“; ausgewählte Forschungsschwerpunkte: (Kriminal-)Rechtspolitik und -anwendungspraxis, Evaluation von Rechtsinstrumenten, Kriminalitätsentwicklung, -statistik und -berichterstattung. 